\title{
Diabetes care in public health facilities in India: a situational analysis using a mixed methods approach
}

This article was published in the following Dove Press journal: Diabetes, Metabolic Syndrome and Obesity: Targets and Therapy

Jaya Prasad Tripathy ${ }^{1,2}$

Karuna D Sagili'

Soundappan Kathirvel ${ }^{1,3}$

Archana Trivedi

Sharath Burugina Nagaraja ${ }^{4}$

Om Prakash Bera ${ }^{1,5}$

Kiran Kumar Reddy'

Srinath Satyanarayana ${ }^{1,2}$

Ashwani Khanna ${ }^{6}$

Sarabjit S Chadha'

'Centre for Operational Research, International Union Against Tuberculosis and Lung Disease, New Delhi, India; ${ }^{2}$ Centre for Operational Research, International Union Against Tuberculosis and Lung Disease, Paris, France;

${ }^{3}$ Department of Community Medicine, Post Graduate Institute of Medical Education and Research, Chandigarh, India; ${ }^{4}$ Department of Community Medicine, Employees State Insurance Corporation Medical College, Post Graduate Institute of Medical Sciences and Research, Bangalore, India;

${ }^{5}$ Bloomberg Data for Health Initiative, Vital Strategies, Mumbai, India;

${ }^{6}$ Department of Health and Family

Welfare, Government of Delhi, New

Delhi, India
Correspondence: Jaya Prasad Tripathy International Union Against Tuberculosis and Lung Disease, The Union South East Asia Office, C-6 Qutub Institutional Area, New Delhi II0016, India

Tel +9l 9478053690

Email ijay.doc@gmail.com
Background: Weak public health systems have been identified as major bottlenecks in providing good quality diabetic care in low- and middle-income countries.

Methodology: The present study assessed diabetic care services at public health facilities across six districts in three states of India using a mixed methods approach. The study described diabetes care services available at public health facilities and identified challenges and solutions needed to tackle them. The quantitative component included assessment of availability of services and resources, whilst the qualitative component was comprised of semistructured interviews with health care providers and persons with diabetes to understand the pathway of care.

Results: A total of 30 health facilities were visited: five tertiary; eight secondary and 17 primary health facilities. Patient clinical records were not maintained at the facilities; the onus was on patients to keep their own clinical records. All had the facility for blood glucose measurement, but HbAlc estimation was available only at tertiary centers. None of the primary health centers in the three states provided HbAlc estimation, lipid examination, or foot care. Lifestyle modification support was available in only a few tertiary facilities. Antidiabetic drugs (biguanides and sulphonyl ureas) were available in most facilities, and given for 14 days. Insulin and statins were available only at secondary and tertiary care centers. Forty-two physicians were interviewed and poor follow-up, patient overload, and lack of specialized training were the major barriers that emerged from the interview responses. A total of 37 patients were interviewed. Patients had to visit tertiary facilities for drugs and routine follow-up, thereby congesting the facilities. There was no formal referral or follow-up mechanism to link patients to decentralized facilities.

Conclusion: There is a wide gap between effective diabetes management practices and their implementation. There should be a greater role of secondary care facilities in follow-up investigations and screening for complications. A holistic diabetic care package with a robust recording and cohort monitoring system and adequate referral mechanism is needed.

Keywords: diabetes mellitus, primary care, screening, noncommunicable disease, mixed methods

\section{Background}

Diabetes mellitus (DM) is one of the most common noncommunicable diseases (NCDs) in the world. According to the International Diabetes Federation estimates, around 425 million people had DM in 2017 and this number is expected to rise to 629 million by $2045 .{ }^{1}$ Around $75 \%$ of persons with DM live in low- and middleincome countries (LMICs). The economic burden of DM globally was estimated to 
be USD $\$ 673$ billion dollars (2015), which was $12 \%$ of the total global health spending. ${ }^{1}$

There are an estimated 69.1 million persons with DM (20-79 years) in India, second only to China. ${ }^{1}$ According to the recent epidemiological surveys, the prevalence of DM in India ranges from $5 \%$ to $17 \% .^{2-4}$ The burden is expected to increase as a result of rapid demographic and lifestyle changes that includes: aging population, increasing urbanization, dietary changes, reduced physical activity and unhealthy behaviors., 3 DM was considered as a disease of the affluent, however, recent epidemiological evidence suggests a rising incidence among the Indian urban middle class and working poor, indicating that all segments of the society are affected by this disease. ${ }^{6}$

The rising burden of the disease and other NCDs prompted Government of India to launch the National Programme for Prevention and Control of Cancers, Diabetes, Cardiovascular Diseases and Stroke (NPCDCS) in 2008 as a pilot in 100 districts. $^{7}$ The package of services to be made available at different levels under the NPCDCS is given in Box 1. The scheme was proposed to be rolled out across the country by 2017 . However, the progress has been dismal with states sitting on huge unspent balance over the last 2 years. The NPCDCS envisages provision of preventive (for high risk groups), promotive (for general population), curative (early diagnosis through screening, management and regular follow-up) and supportive services (palliative care and rehabilitation) in Cancer, Diabetes, Cardio-Vascular Diseases (CVD) and Stroke through the public health system. Since the inception of the program there has been no systematic evaluation of the services rendered under the program. A survey conducted in Mumbai among 2,269 persons with DM found that around half of the patients had poor glycemic control (HbAlc $>2 \%$ points above upper limit of normal) and over $54 \%$ had diabetes-related complications. ${ }^{8}$ Few other studies had shown that poor quality of medical care especially for those requiring chronic treatment was associated with noncompliance to the treatment regimen and discontinuation of care. ${ }^{9}$ Among persons with DM, discontinuation of care was found to be associated with worse glycemic control $^{10}$ and an increased risk of complications. ${ }^{11}$ Weak public health systems have been identified as major bottlenecks in providing quality diabetic care in LMICs, including India. ${ }^{12,13}$

Box I Packages of services to be made available at different levels under NPCDCS in India

\begin{tabular}{|c|c|}
\hline Health Facility & Packages of services \\
\hline Sub-center & $\begin{array}{l}\text { I. Health promotion for behavior change } \\
\text { 2. "Opportunistic" screening using BP measurement and blood glucose by strip method } \\
\text { 3. Referral of suspected cases to CHC }\end{array}$ \\
\hline CHC & $\begin{array}{l}\text { 1. Prevention and health promotion including counseling } \\
\text { 2. Early diagnosis through clinical and laboratory investigations (common lab investigations: blood sugar, lipid profile, ECG, } \\
\text { ultrasound, } X \text { ray, etc.) } \\
\text { 3. Management of common CVD, diabetes and stroke cases (outpatient and inpatients.) } \\
\text { 4. Home-based care for bed ridden chronic cases } \\
\text { 5. Referral of difficult cases to district hospital/higher health care facility }\end{array}$ \\
\hline District hospital & $\begin{array}{l}\text { I. Early diagnosis of diabetes, CVDs, stroke } \\
\text { and cancer } \\
\text { 2. Investigations: blood sugar, lipid profile, kidney function test (KFT), liver function test (LFT), ECG, ultrasound, X ray, } \\
\text { colposcopy, mammography, etc. (if not available, will be outsourced) } \\
\text { 3. Medical management of cases (outpatient, inpatient and intensive care) } \\
\text { 4. Follow-up and care of bed ridden cases } \\
\text { 5. Day care facility } \\
\text { 6. Referral of difficult cases to higher health care facility } \\
\text { 7. Health promotion for behavior change }\end{array}$ \\
\hline $\begin{array}{l}\text { Tertiary care } \\
\text { centre }\end{array}$ & $\begin{array}{l}\text { In addition to the services above, comprehensive cancer care including prevention, early detection, diagnosis, treatment, } \\
\text { minimal access surgery, after care, palliative care, rehabilitation }\end{array}$ \\
\hline
\end{tabular}

Abbreviations: CHC, Community Health Centre; CVD, cardiovascular disease; ECG, electrocardiogram; BP, blood pressure; NPCDCS, National Programme for Prevention and Control of Cancer, Diabetes, Cardiovascular Diseases and Stroke. 
Against this background, we conducted this study to assess the diabetic care services provided at public health facilities across three states (Delhi, Karnataka and Maharashtra) in the country using a mixed methods approach. The objectives of the assessment were to:

1. describe the available diabetic care services at public health facilities

2. identify the challenges and interventions that are needed to strengthen diabetic care services.

\section{Methods}

\section{Study design}

We employed a sequential mixed methods study design. The quantitative component included assessment of the availability of protocols, tools/equipments, drugs, diagnostics and human resources for screening, diagnosing and managing DM by retrospective review of hospital records, while the qualitative component comprised of semi-structured interviews with health care providers and persons with DM.

\section{Study setting}

In India, primary health care is provided through a network of sub-centers (SCs) and primary health centers (PHCs). The SC is manned by Auxiliary Nurse Midwife/ Multipurpose Health worker and serves a population of 5,000 . The PHC, staffed by Medical Officer and other paramedical staff serves every 30,000 population. There are 5-6 SCs under every PHC. Patients from PHCs are referred to secondary level facilities (district level hospital/ community health centres (CHCs)) for better management. Tertiary health care refers to the third tier of health system, in which specialized consultative care is provided usually on referral from primary and secondary level facilities. Tertiary care is provided by medical colleges and advanced medical research institutes.

\section{Study sites}

This study was conducted in six districts across three states, namely, Delhi (East and Central districts), Karnataka (Tumkur and Kolar) and Maharashtra (Amaravati rural and Amravati urban) state. The study districts were selected based on convenience and operational feasibility. In each district, one tertiary hospital (medical college) except Amravati urban, 1-2 sub-district level hospitals/CHCs and 2-4 PHCs (one/two from each sub-district hospital/CHC geographical coverage area) were selected from a list of such health facilities randomly. The number of health facilities was based on operational feasibility.

\section{Study participants}

At each health facility, the health care providers (1-3 treating physicians and one pharmacist) and persons with DM were interviewed using a semi-structured interview guide. The physicians were either general practitioners (in $\mathrm{PHCs} / \mathrm{CHCs}$ ) or faculty in the department of medicine or endocrinology (in tertiary health facilities). The persons with DM (2-4 numbers) were identified randomly among those waiting for doctor's consultation and were interviewed after completion of their consultation with the doctor. The numbers of interviews were based on the saturation of findings. Participants were recruited until no new relevant information pertaining to the major theme was being obtained. Written informed consent was obtained prior to the interviews.

\section{Study period}

The study was conducted between July and September 2016.

\section{Data collection}

There were two dedicated teams comprising of a senior researcher/consultant and a trained interviewer in qualitative research from The Union office, New Delhi deployed for data collection. The teams were trained for 2 days on the process of data collection. The interview guides and the data collection instruments were developed during a consultative workshop held for primary stakeholders. The instruments were pilot tested before data collection in health facilities other than those included in this study. The tools are attached as annexures 1-4.

\section{Quantitative data}

Aggregate data were collected from the hospital records and drug stock registers. An observation checklist was used to assess the availability of manpower, equipments, drugs, diagnostics and other services.

\section{Qualitative data}

Personal interviews with health care providers and persons with DM were conducted using a semi-structured questionnaire to explore the nature of diabetic care services in public health facilities. The interviews were conducted at a time and place convenient to the participants using an 
interview guide. Each interview lasted around 20 mins. Only the researcher and the participant were present during the interviews. The investigator held one-to-one interviews after explaining the study purpose and after obtaining their consent to participate. Responses to openended questions were manually noted down and transcribed later. After each interview, the summary of the interviews was read back to the participants to ensure participant validation.

\section{Data analysis \\ Quantitative data}

Quantitative data were entered into a pre-designed excel sheet and shared among study investigators via dropbox (file sharing tool) and email. It was cross-checked for completeness and used for analysis. Quantitative data (availability of drugs, equipment and services) were presented in the form of proportions by type of health facility.

\section{Qualitative data}

The transcripts and notes were transcribed and analyzed manually by the primary investigators. ${ }^{14,15}$ Codes were generated and similar codes were combined into themes. ${ }^{14}$ The themes have been described and English translations of some of the transcribed quotes have been included. The translation from the local language (Hindi) to English was done by JPT after transcription of the quotes.

The transcripts were analyzed to understand various links in their pathway of accessing diabetic care at different stages of the disease - initial diagnosis, drugs, followup investigations and complication screening. A flow chart was created for each patient tracing their pathway of care starting from the onset of symptoms and initial diagnosis to current care.

\section{Ethics}

Ethical approval was obtained from the Union Ethics Advisory Group, Paris, France. Permission and support to conduct the study was obtained from the State and District NCD Nodal Officer in Karnataka and Delhi and the Office of Joint Director, Directorate of Health Services in Maharashtra. Written informed consent was obtained from the participants before carrying out the interviews.

\section{Results}

\section{Quantitative data}

General information about the public health facilities: A total of 30 health care facilities were assessed of which five were tertiary care facilities; eight were secondary and 17 primary health facilities. The details of the health facilities have been summarized in Table 1 .

\section{Recording and reporting of diabetic services}

The diabetic status of patients was recorded in the general outpatient registers in more than half of the PHCs $(10 / 17$, $59 \%)$. In some of the primary $(6 / 17,35 \%)$ and tertiary $(2 / 5,40 \%)$ care facilities and all secondary level hospitals $(8 / 8,100 \%)$, there was a separate NCD register in which detailed information about persons with diabetes who visited every day were recorded. Besides sociodemographic and clinical profile, information related to diagnosis, other investigations and treatment of diabetes in the initial and follow-up visits are recorded in the NCD register. The details of patients availing the laboratory facilities and

Table I General information, recording and reporting mechanism for diabetes mellitus in public health facilities in three states of India, 2016

\begin{tabular}{|l|l|l|l|}
\hline Level of health facility & Tertiary (\%) & Secondary (\%) & Primary (\%) \\
\hline Number of facilities visited & 5 & 8 & 17 \\
Number of beds for inpatient care at the visited facilities (range) & $300-1,800$ & $30-200$ & $0-5$ \\
Daily OPD load (range) & $1,500-6000$ & $180-2,500$ & $35-200$ \\
Availability of special clinic days for DM & $5(100)$ & $3(38)$ & $5(29)$ \\
Daily DM load/on special clinic days (range) & $45-400$ & $15-100$ & $5-30$ \\
DM recorded in general OPD register & $0(0)$ & $0(0)$ & $10(59)$ \\
Recorded in separate NCD register & $1(40)$ & $8(100)$ & $6(35)$ \\
Electronic patient record maintained for DM outpatients & $3(20)$ & $0(0)$ & $0(0)$ \\
DM patient case file/record maintained at the hospital & $2(40)$ & $1(12)$ & $0(0)$ \\
Patient card/health card with the DM patient & $2(25)$ & 24 \\
\hline
\end{tabular}

Notes: Numbers indicate number of facilities; numbers within parentheses indicate percentage denominator being total number of facilities visited in that category Abbreviations: DM, diabetes mellitus; OPD, outpatient department; NCD, noncommunicable disease. 
pharmacy were available in the laboratory and drug registers. However, the information in these registers were not linked to each other.

At the health facilities in Maharashtra, the patients were issued NCD cards which had basic information of the patients, diseases, drugs dispensed, results of laboratory investigations, follow-up visits and health education messages. One tertiary care facility in Delhi had maintained electronic patient record for each patient and followed up patients regularly. Patient case files (hardcopy) were also maintained at this center. Tertiary care hospitals in Karnataka maintained clinical files of persons with diabetes at the facility. Apart from these centers, the onus of maintaining their medical records was on the patients (Table 1). There was no mechanism or system for follow-up of patients.

\section{Availability of diagnostic and monitoring facilities}

All health centers had facilities for blood glucose measurement using glucometer. Estimation of glycosylated hemoglobin $(\mathrm{Hb} 1 \mathrm{Ac})$ was available at all tertiary care facilities and one secondary health facility. Electrocardiogram, blood lipid profile and foot care was available in all tertiary and most (75\%) secondary level health facilities. Ophthalmic fundus examination was also available in all tertiary and 7 of $8(88 \%)$ secondary hospitals. Echocardiogram and facilities for dialysis was available only in tertiary hospitals. None of the PHCs in any of the three states had the facility for HbAlc estimation, lipid profile examination and foot care services (Table 2).

\section{Availability of drugs}

The oral hypoglycemic agents (OHAs) such as biguanide (metformin) and at least one of the sulphonyl ureas (like glimepiride, glybenclamide and gliclazide) were available at all health facilities. Insulin and statins were available at all tertiary care centers and in some secondary level hospitals (38-50\%). Anti-hypertensive drugs like atenolol/

Table 2 Availability of laboratory investigations, drugs and lifestyle modification support in public health facilities in three states of India, 2016

\begin{tabular}{|c|c|c|c|}
\hline Level of health facility & Tertiary & Secondary & Primary \\
\hline Number of facilities visited & 5 & 8 & 17 \\
\hline \multicolumn{4}{|l|}{ Laboratory investigations } \\
\hline Glucometer/blood glucose & $5(100)$ & $8(100)$ & $17(100)$ \\
\hline $\mathrm{HbAlc}$ & $5(100)$ & $I(12)$ & $0(0)$ \\
\hline Lipid screening & $5(100)$ & $6(75)$ & $0(0)$ \\
\hline Fundus examination & $5(100)$ & $7(88)$ & $0(0)$ \\
\hline Urine examination & $5(100)$ & $8(100)$ & $12(71)$ \\
\hline Foot care & $5(100)$ & $6(75)$ & $0(0)$ \\
\hline ECG & $5(100)$ & $6(75)$ & $0(0)$ \\
\hline Drugs & & & $0(0)$ \\
\hline Glimepiride & $5(100)$ & $6(75)$ & $8(47)$ \\
\hline Glybenclamide & $0(0)$ & $5(62)$ & $8(47)$ \\
\hline Glyclazide & $2(40)$ & $2(25)$ & $0(0)$ \\
\hline Metformin & $5(100)$ & $7(88)$ & $14(82)$ \\
\hline Gliptin & $3(60)$ & $I(I 2)$ & $0(0)$ \\
\hline Insulin & $5(100)$ & $4(50)$ & $0(0)$ \\
\hline Atenolol/Metoprolol & $5(100)$ & $8(100)$ & $15(88)$ \\
\hline Amlodipine & $5(100)$ & $6(75)$ & $13(77)$ \\
\hline Losartan & $2(40)$ & $0(0)$ & $0(0)$ \\
\hline Enalapril & $4(80)$ & $I(12)$ & $3(18)$ \\
\hline Atorvastatin & $5(100)$ & $3(38)$ & $0(0)$ \\
\hline Antidiabetic drugs given for how many days (range) & $10-30$ & $7-30$ & $7-30$ \\
\hline \multicolumn{4}{|l|}{ Lifestyle modification support } \\
\hline Dietary counselling & $5(100)$ & $6(75)$ & $0(0)$ \\
\hline Smoking cessation counselling & $2(40)$ & $2(25)$ & $0(0)$ \\
\hline Yoga/physiotherapy/exercise & $2(40)$ & $2(25)$ & $0(0)$ \\
\hline Patient education on complications and their prevention & $2(40)$ & $I(12)$ & $2(12)$ \\
\hline
\end{tabular}

Notes: Numbers indicate number of facilities; numbers within parentheses indicate percentage, denominator being total number of facilities visited in that category. Abbreviations: ECG, electrocardiogram; ECHO, echocardiogram. 
metoprolol were available at all the health facilities. Angiotensin-converting enzyme (ACE) inhibitors were available only in tertiary health facilities in Delhi. The OHAs and insulin were given to the patients for about 14 days in Delhi and Maharashtra and for about 30 days in Karnataka (Table 2).

\section{Lifestyle modification support}

Dietary counseling service was available in all tertiary facilities and most $(6 / 8,75 \%)$ of the secondary level facilities. However, smoking cessation counseling, yoga/exercise and patient education were available in few tertiary $(2 / 5,40 \%)$ and secondary care facilities (12-25\%) (Table 2).

\section{Qualitative data}

\section{Interviews with physicians}

A total of 42 physicians (mostly internal medicine) from different levels of health care facilities in three states were interviewed using a semi-structured guide to understand the operational gaps in service delivery and their recommendations to address them. The responses have been summarized below.

Most of the doctors at primary care facilities did not receive any specialized training in diabetes. There was no written protocol for screening and management of diabetes in most of the facilities. There is no universal blood glucose screening criterion being followed. The health care providers reported that they screen individuals based on some arbitrary age cutoff (30 years or $\geq 45$ years), presence of obesity, pregnancy, family history or those with symptoms suggestive of the disease.

The following themes emerged from the physician's interviews which have been described along with verbatim quotes.

\section{Poor follow up of patients}

Although most of the doctors advise regular follow-up every 15-30 days, they also shared their concern that it is difficult to keep track of every patient due to lack of patient follow-up details and more importantly, lack of time to maintain patient case records due to deficient manpower. One of the physicians said:

I have to see these many patients daily. There is no time available to maintain follow-up records and using the same at every visit. [Physician, female, 56 years old]

\section{Patient overload}

Physicians at the tertiary care facilities were overloaded with patients in the tune of 200-500 persons with diabetes daily. Some of them suggested reducing the patient load by a mechanism of referral and linkage with the peripheral facilities. Few others suggested the alternative option of increasing the manpower to handle this load of patients.

Many patients come here for drugs only, there should be a referral mechanism so that they get drugs from other centers.

Only those patients who require specialised diabetic care or follow up for complications should come to a tertiary care facility. [Physician, male, 42 years old]

Lack of manpower was also cited as one of the main challenges in delivering good quality diabetic care. There is gross doctor: patient imbalance with each doctor serving more than 400 patients daily in a tertiary care setup. Even in the primary care setting a single doctor has to cater to more than 100 patients daily along with administrative, managerial and other ad-hoc responsibilities.

If we have to see 200 patients per day, then we can devote only $2-3$ mins per patient, there is shortage of doctors.

[Physician, female, 50 years old]

\section{Lack of training}

Lack of specialized training among physicians mostly at primary level health facilities was a limitation. Trained manpower at primary level is required to deliver standard diabetic care and establish and maintain a referral linkage system with the secondary/tertiary level.

The operational issues and suggestions/solutions as described by the respondents (physicians) have been summarized in Table 3.

\section{Interviews with persons with diabetes}

A total of 37 patients were interviewed at 12 facilities from different levels. Most of the patients reported overcrowding, long waiting queues and inadequate care as the major barriers to receiving healthcare at public health facilities. At the primary level, patients visited the hospital for drugs or routine follow-up. However, at secondary and tertiary level, they presented for investigations and routine follow-up, complication screen and some even came for drugs only. Most of them were on OHAs. More than half had tried alternative medicine such as Ayurveda, Unani, Yoga, etc. Almost all of them got medicines free of cost from the facility. However, medicines were given for 2 weeks and patients felt that they 
Table 3 Operational issues identified and solutions as suggested by physicians in public health facilities of three states in India, 2016

\begin{tabular}{|l|l|}
\hline Operational issue identified & Solution proposed \\
\hline No regular F/U or tracking of patients & Maintenance of patient records electronically or as case files \\
Patient overload, Too less time for each & Patients should not come to tertiary/secondary facilities for drugs only, linkage with primary care \\
patient & facilities for drugs and routine follow-up, drug dispensing for one month \\
Lack of specialists/manpower & Training existing manpower-doctors/paramedical staff in diabetic care and management \\
No dedicated team for diabetic care & Specialised training courses for existing manpower to build a team \\
No system of back referral and linkage with & Develop a mechanism of referral and linkage with primary care facilities along the lines of DOTS in \\
primary health facilities & RNTCP \\
Difficult to manage complications at PHC & Equip PHCs with diagnostic and follow-up laboratory investigations, provide specialised training on \\
Lack of lab investigations at PHC & diabetic care to manpower at PHC \\
Shortage of medicine at PHC & Efficient procurement and indenting mechanism, real time drug procurement system \\
\hline
\end{tabular}

Abbreviations: PHC, primary health center; F/U, follow-up; RNTCP, Revised National Tuberculosis Control Programme; DOTS, Directly Observed Treatment Short Course.

have to come quite often. In order to avoid repeated hospital visits, some patients also purchased medicines from private pharmacy.

We have to keep coming, stand in the queue only for 2week medicines. [58 year old male patient]

If medicines are given for long duration, it will be good, who likes visiting a hospital frequently. [46 year old female patient]

If medicines are given for a month, my expenditure can be reduced. [60 year old male patient]

Most patients replied that they hardly maintain records of past medical care related to diabetes. Some of them had some OPD slips only. "Whatever it is, it is available in this slip, where do I find the old slips now." [50 year old male patient]

Three of 37 patients interviewed were on insulin. Although insulin was available in secondary/tertiary level facilities, patients reported that instructions on how to store, use and dispose insulin and the syringes were not given appropriately.

Patients who visited private providers to receive care cited financial constraint as a major barrier to accessing diabetes care.

\section{Pathways of care}

Transcripts of interviews with 20 patients (complete information was available in these patients only) were assessed to analyze the pathways of care. Among those interviewed, $10(50 \%)$ were males. The average age of the patients was 51 years. Majority of these patients were diagnosed at a tertiary level facility $(8,40 \%)$ or a private provider $(8$, $40 \%$ ) following which most of them $(9,45 \%)$ continued to receive routine care at a tertiary level which partially explains the reasons for overcrowding at these centers. A substantial proportion of patients $(8 / 20,40 \%)$ visited private care providers for diagnosis of diabetes and follow-up investigations.

The tertiary level facilities and the private providers were approached for investigations such as HbAlc and for screening of complications, understandably so because of the nonavailability of the service at other levels of facilities. The flowchart depicts a greater role of tertiary care facilities in provision of diabetic care and minimal involvement of primary and secondary level facilities. (Figure 1)

\section{Discussion}

It is one of the few mixed methods studies assessing diabetic care services provided at public health care facilities in India. The study showed uninterrupted supply of drugs (both anti-diabetic and anti-hypertensives) at all levels of care and adequate laboratory infrastructure especially in the secondary and tertiary facilities which was quite encouraging. On the other hand, the study findings also revealed significant gaps in diabetic care delivery at public health facilities. There was sparse or no information regarding diagnosis, treatment, follow-up, associated comorbidities and monitoring of treatment outcomes for persons with DM. A qualitative study in South India revealed various constraints that patients face in accessing diabetes care. These included financial hardship, negative attitudes and inadequate communication by health care providers and a fragmented primary health care system offering inadequate care, similar to the findings of the present study. ${ }^{12}$ Similarly, population-based studies in 


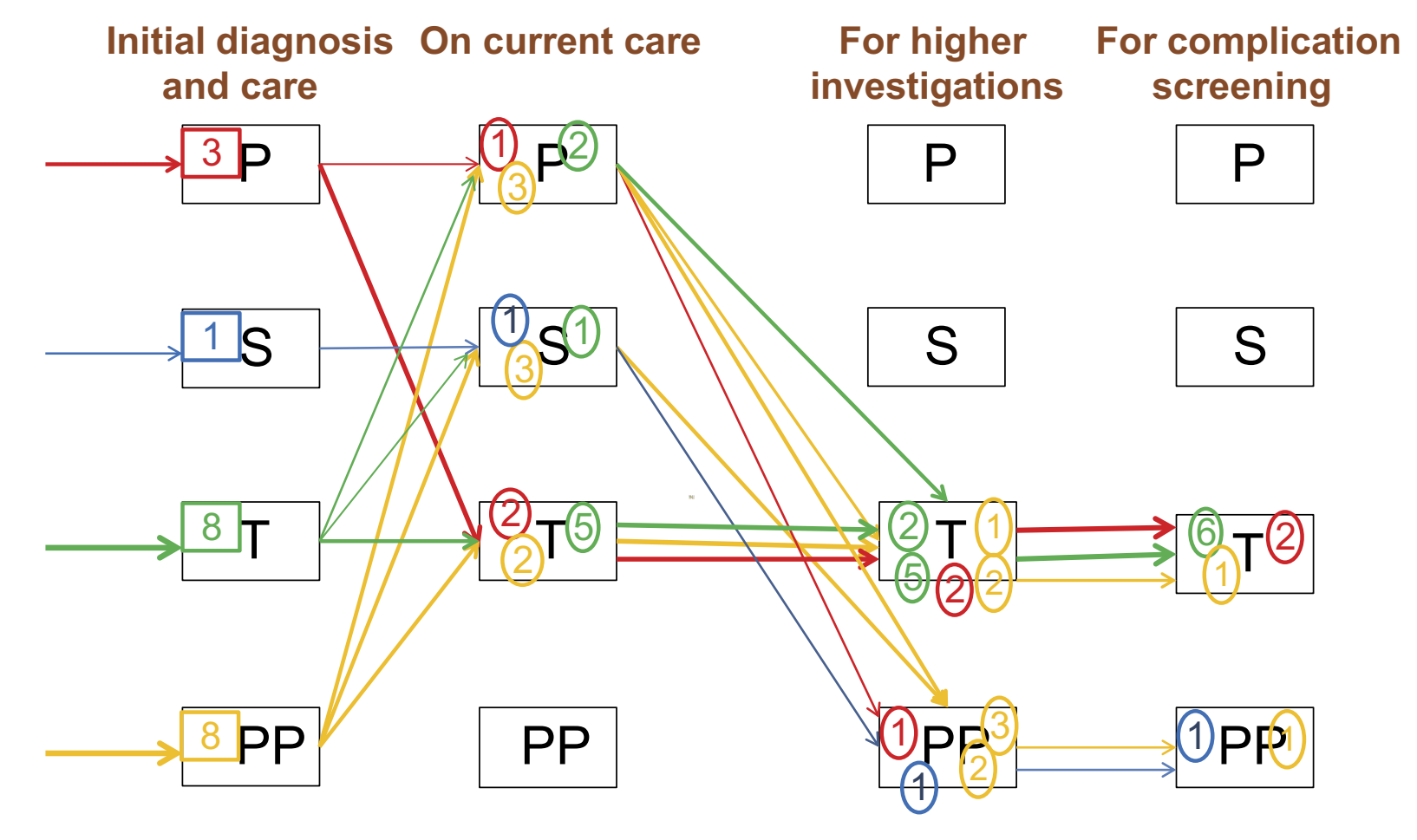

\begin{abstract}
$\mathrm{P}=$ Primary level facility; $\mathrm{S}=\mathrm{Secondary}$ level facility; $\mathrm{T}=$ Tertiary level facility; $\mathrm{PP}=$ Private Practitioner; numbers within circles/boxes indicate number of diabetic patients; boldness of the arrow indicate number of patients
\end{abstract}

Figure I Pathways to diabetic care for 20 patients.

both northern and southern parts of India have shown wide gap between practice recommendations and delivery of diabetes care leading to poor glycemic control and adverse clinical outcomes. ${ }^{16,17}$

The findings of our study have the following programmatic implications. First, the inability of the system to convert the available public health data into useful information calls for the adoption of a "cohort" strategy of recruiting the patient and following them up at regular intervals. The Revised National TB Control Programme (RNTCP) has efficiently adopted the cohort monitoring system and is successful in generating robust recording and reporting of patients enrolled in the program. ${ }^{18}$ The same was suggested by Harries et al for better monitoring of care and management of chronic diseases. ${ }^{19}$ The "cohort" monitoring system in other resource limited settings such as Malawi and Jordan for chronic diseases such as DM and hypertension has been found to be successful. They have used an electronic medical record system that allows regular follow-up of patient cohorts and yields useful information about their programmatic and management outcomes, quality of care and disease control, development of complications, etc. ${ }^{20-22}$ Thus, we propose an ICT (information and communication technology) enabled recording and reporting system for registering all persons with DM and maintaining their records. These records will provide information on patient management, compliance to various management decisions, status of glycemic control and key laboratory and clinical parameters. It will also aid in clinical decision-making and provide patient education.

Second, qualitative data analysis suggests that patient overload at the tertiary care facilities is an hindrance to record keeping and timely follow-up of patients which is quite resource intensive and time constraining. The patient care pathway also shows that apart from follow-up investigations and screening for complications, most patients also go to tertiary facilities for routine care and drugs. This requires linkage with peripheral health facilities and their strengthening to decentralize services in order to decongest tertiary facilities. There should be a greater role of secondary care facilities in follow-up investigations 
(HbA1c) and screening for complications. Routine care and provision of drugs should be taken care of at the primary care level along the lines of Directly Observed Treatment Short Course (DOTS) in the RNTCP where patients are referred to their nearest DOT center for treatment, which is a decentralized peripheral unit and are referred to a higher center in case of any complications or follow-up.

Third, though a large number of diabetic patients are being treated at the public health facilities, there were no standard protocols for providing care to persons with diabetes. Based on their prior knowledge and cumulative experience during their graduation and post-graduation, health care providers had their own criteria for screening, diagnosing and managing these patients with the existing resources. Hence, a standard diabetic care and management protocol which is acceptable to all providers needs to be implemented.

Fourth, some of the essential laboratory investigations like glycosylated hemoglobin, fundus examination and drugs (insulin, ACE inhibitors) were available only at tertiary and few secondary health facilities. The needy patients requiring these tests/drugs were expected to physically visit these facilities in order to avail those services. There was no formal referral or follow-up mechanism to link patients to these services and facilities. Similarly, there was no formal mechanism of linking complicated cases to secondary and tertiary health facilities and referral of un-complicated cases at secondary and tertiary health facilities to primary health care facilities. As a result, secondary and tertiary care facilities were over-crowded with many patients visiting these centers to get their fortnightly quota of anti-diabetic medicines - a service that could easily be fulfilled at the primary health facilities. This requires strengthening of the primary care facilities by making key investigations and drugs available and a robust referral and back-referral mechanism between different levels of care. It will ensure that the patient receives appropriate care at the right level with minimal hassles.

Fifth, sub-optimal health education was provided to patients at health facilities. Advice and patient support for dietary modification, physical activity and tobacco cessation were limited to advice given by the treating medical doctors as a part of routine OPD care. There was no mechanism to provide customized or structured counseling to patients and track patients' response to these advice. There were no systems in place to maintain individual patient records at health facilities to document the details of patients' treatment and their response to therapy and the onus of maintaining one's records were on the patients. As a result of these lacunae, the quality of care that patients receive is largely unknown. Therefore, we propose a "standardized package of care", including screening, essential investigations and drugs, regular follow-up care and patient education and counseling as outlined in the NPCDCS guidelines that can be implemented at all primary health facilities with adequate linkages with secondary and tertiary health care facilities.

There were some limitations in this study. First, owing to a lack of cohort monitoring and reporting of registered persons with DM, clinical outcomes of patients and attrition from care could not be assessed. Second, selection of health facilities was not at random which might affect the generalisability of findings, although all three levels of facilities were covered. Third, the study was only limited to public health facilities, thus excluding the private providers who play a crucial role in managing persons with diabetes in the community. On the other hand, a mixed methods approach used in this study added robustness to the evidence generated with qualitative data substantiating the quantitative information.

\section{Conclusion}

To conclude, there is a wide gap between effective diabetes management practices and their implementation, at various levels of health facilities. The recommended strategies for diabetic care like self-management education, nutrition counseling, exercise prescription and screening are sub-optimally delivered. Strengthening the recording and quarterly reporting is of paramount importance to monitor the quality of diabetic care services provided at the health facilities. A holistic diabetic care package with evidence-based practices and adequate referral mechanism is the need of the hour.

\section{Abbreviations list}

PHC, primary health center; CHC, community health Center; ICT, information and communication technology; NCD, noncommunicable disease; RNTCP, Revised National TB Control; DM, diabetes mellitus program; CVD, cardiovascular disease; OPD, outpatient department; LMIC, low- and middle-income countries; ACE, angiotension converting enzyme; OHA, oral hypoglycemic agent. 


\section{Availability of data}

Data are available with the corresponding author (JPT) and will be available on request at the following email address: ijay.doc@gmail.com.jtripathy@theunion.org.

\section{Ethics}

Ethical approval was obtained from the Union Ethics Advisory Group, Paris, France. Permission and support to conduct the study was sought from the state authorities in all three states. Written informed consent was obtained from the participants before carrying out the interviews.

\section{Acknowledgments}

We thank the Department for International Development (DFID), UK, for funding the Global Operational Research Fellowship Programme at the International Union Against Tuberculosis and Lung Disease (The Union), Paris, France, in which Jaya Prasad Tripathy and Srinath Satyanarayana work as Senior Operational Research Fellows. The study was funded by Lilly MDR-TB Partnership.

\section{Author contributions}

JPT, KDS, and SS were involved in conceptualization of the idea. All authors contributed towards data analysis, drafting and critically revising the paper, gave final approval of the version to be published, and agreed to be accountable for all aspects of the work.

\section{Disclosure}

The authors report no competing interests in this work.

\section{References}

1. International Diabetes Federation. IDF Diabetes Atlas, 8th edition. Brussels, Belgium: International Diabetes Federation; 2017.

2. Mohan V, Sandeep S, Deepa R, Shah B, Varghese C. Epidemiology of type 2 diabetes: Indian scenario. Indian J Med Res. 2007;125:217-230.

3. Anjana RM, Pradeepa R, Deepa M, et al. Prevalence of diabetes and prediabetes (impaired fasting glucose and/or impaired glucose tolerance) in urban and rural India: phase I results of the Indian Council of Medical Research-INdia DIABetes (ICMR-INDIAB) study. Diabetologia. 2011;54:3022-3027. doi:10.1007/s00125-010-2040-1

4. Ramachandran A, Snehalatha C, Kapur A, et al. High prevalence of diabetes and impaired glucose tolerance in India: national urban diabetes survey. Diabetologia. 2001;44:1094-1101. doi:10.1007/ s001250100008

5. Ebrahim S, Kinra S, Bowen L, et al. The effect of rural-to-urban migration on obesity and diabetes in India: a cross-sectional study. PLoS Med. 2010;7:e1000268. doi:10.1371/journal.pmed.1000268
6. Mendenhall E, Shivashankar R, Tandon N, Ali MK, Narayan KMV, Prabhakaran D. Stress and diabetes in socioeconomic context: a qualitative study of urban Indians. Soc Sci Med. 2012;75:25222529. doi:10.1016/j.socscimed.2012.09.040

7. National programme for prevention and control of cancer, diabetes, cardiovascular diseases and stroke operational guidelines. directorate general of health services, ministry of health \& family welfare government of India. [Internet]. Available from: http:/health.bih. nic.in/Docs/Guidelines/Guidelines-NPCDCS.pdf. Accessed July 21, 2016.

8. Raheja BS, Kapur A, Bhoraskar A, et al. DiabCare Asia-India study: diabetes care in India-current status. J Assoc Physicians India. 2001;49:717-722.

9. Vuori H. Patient satisfaction-does it matter? Qual Assur Health Care. 1991;3(3):183-189.

10. Jacobson AM, Adler AG, Derby L, Anderson BJ, Wolfsdorf JI. Clinic attendance and glycemic control. Study of contrasting groups of patients with IDDM. Diabetes Care. 1991;14:599-601. doi:10.2337/ diacare.14.7.599

11. Hammersley MS, Holland MR, Walford S, Thorn PA. What happens to defaulters from a diabetic clinic? $\mathrm{Br}$ Med J (Clin Res Ed). 1985;291:1330-1332. doi:10.1136/bmj.291.6505.1330

12. Bhojani U, Mishra A, Amruthavalli S, et al. Constraints faced by urban poor in managing diabetes care: patients' perspectives from South India. Glob Health Action. 2013;6:22258. doi:10.3402/gha. v6i0.22258

13. Venkataraman K, Kannan AT, Mohan V. Challenges in diabetes management with particular reference to India. Int $J$ Diabetes Dev Ctries. 2009;29:103-109. doi:10.4103/0973-3930.50711

14. Creswell J, Plano Clark V. Designing and Conducting Mixed Methods Research. London (United Kingdom): Sage Publications Ltd; 2007:142-145.

15. Kvale S. Doing Interviews. London (United Kingdom): SAGE Publications; 2007.

16. Nagpal J, Bhartia A. Quality of diabetes care in the middle- and high-income group populace: the Delhi Diabetes Community (DEDICOM) survey. Diabetes Care. 2006;29:2341-2348. doi: $10.2337 / \mathrm{dc} 06-0783$

17. Ramachandran A, Mary S, Sathish CK, et al. Population based study of quality of diabetes care in southern India. $J$ Assoc Physicians India. 2008;56:513-516.

18. Central TB Division Ministry of Health and Family Welfare. TB India report 2018 revised national tuberculosis programme annual status report [Internet]. Available from: http://tbcindia.nic.in/showfile.php?lid=3180. Accessed December $18,2016$.

19. Harries AD, Jahn A, Zachariah R, Enarson D. Adapting the DOTS framework for tuberculosis control to the management of noncommunicable diseases in Sub-Saharan Africa. PLoS Med. 2008;5:e124. doi:10.1371/journal.pmed.0050124

20. Khader A, Farajallah L, Shahin Y, et al. Cohort monitoring of persons with diabetes mellitus in a primary healthcare clinic for palestine refugees in Jordan. Trop Med Int Health. 2012;17:1569-1576. doi:10.1111/j.1365-3156.2012.03097.x

21. Khader A, Farajallah L, Shahin Y, et al. Cohort monitoring of persons with hypertension: an illustrated example from a primary healthcare clinic for palestine refugees in Jordan. Trop Med Int Health. 2012;17:1163-1170. doi:10.1111/j.1365-3156.2012.03048.x

22. Manjomo RC, Mwagomba B, Ade S, et al. Managing and monitoring chronic non-communicable diseases in a primary health care clinic, Lilongwe, Malawi. Public Health Action. 2016;6:60-65. doi:10.5588/ pha.16.0003 


\section{Publish your work in this journal}

Diabetes, Metabolic Syndrome and Obesity: Targets and Therapy is an international, peer-reviewed open-access journal committed to the rapid publication of the latest laboratory and clinical findings in the fields of diabetes, metabolic syndrome and obesity research. Original research, review, case reports, hypothesis formation, expert opinion and commentaries are all considered for publication. The manuscript management system is completely online and includes a very quick and fair peer-review system, which is all easy to use. Visit http://www.dovepress.com/testimonials.php to read real quotes from published authors.

Submit your manuscript here: https://www.dovepress.com/diabetes-metabolic-syndrome-and-obesity-targets-and-therapy-journal 